\title{
Adesão e Não-Adesão ao Tratamento Farmacológico para Depressão ${ }^{1}$
}

\author{
Marines de Fátima Cunha ${ }^{2}$ \\ Rita de Cássia Gandini \\ Universidade Federal de Uberlândia
}

\begin{abstract}
RESUMO - O presente estudo objetivou compreender aspectos relacionados à adesão e a não-adesão ao tratamento farmacológico para depressão, envolvendo usuários e ex-usuários de um serviço público de saúde mental. Foram entrevistados, individualmente, 24 pacientes (12 aderentes e 12 não aderentes ao tratamento medicamentoso para depressão). Por meio da análise de conteúdo das entrevistas, identificou-se que o fenômeno da adesão/não-adesão estaria inter-relacionado a aspectos intrapessoais, interpessoais e ao contexto do tratamento do paciente. Os resultados fomentaram reflexões acerca do papel de equipes de saúde mental no incremento da adesão ao tratamento, bem como da necessidade de uma maior consideração do indivíduo, da família e do atendimento prestado pela instituição.
\end{abstract}

Palavras-chave: adesão ao tratamento; não-adesão ao tratamento; depressão.

\section{Compliance and Non Compliance to the Pharmacological Treatment for Depression}

\begin{abstract}
The present study aimed to understand the aspects related to compliance and non compliance to the pharmacological treatment for depression, with users and former-users of a mental-health public service. Twenty four patients were interviewed individually (12 adherent and 12 non-adherent to the pharmacological treatment for depression). Through interviews content analysis, it was identified that the compliance and non-compliance phenomenon would be inter-related to intrapersonal aspects, interpersonal aspects, and to the context of the patient's treatment, The results contributed to reflections concerning to the role of mental health teams in enhancing treatment compliance, as well as to the need of a greater consideration of the individual, the family and the community, and the assistance provided by the institution.
\end{abstract}

Keywords: compliance; non-compliance; depression.

A depressão tem sido avaliada como um transtorno com alta prevalência, possibilidade de recorrência e cronicidade (Fleck \& cols., 2003; Hexsel, 2004), que pode afligir parcela considerável da população, independente de sexo, idade ou etnia (Souza, Fontana \& Pinto, 2005). Essa doença vem sendo apontada como um grave problema de saúde pública, relacionado a elevados custos sociais e risco de suicídio (Hexsel, 2004; Machado, 2003). Segundo dados do relatório da World Health Organization (2001), as perturbações depressivas unipolares, dentre todas as doenças, ocupariam o quarto lugar, em se considerando a geração de encargos, respondendo por 4,4\% do total de AVAI (Ano de Vida Ajustado por Incapacidade) e sendo a principal causa de AVI (Anos Vividos com Incapacidade).

Desde 1950, consistentes evidências têm sido obtidas acerca da eficácia do tratamento farmacológico nas depressões. Segundo Lima, Sougey e Vallada-Filho (2004), os medicamentos antidepressivos têm reduzido a morbidade e melhorado o desfecho clínico da doença. Assim, as medicações antidepressivas têm sido consideradas eficazes no tratamento de todos os graus de depressão, melhorando os

1 Artigo baseado na Dissertação de Mestrado da primeira autora, desenvolvida sob orientação da segunda autora.

2 Endereço para correspondência: Av. Batalhão Mauá, 1482. Centro. Araguari, MG. CEP 38440-210.E-mail: marinescunha@yahoo.com.br. sintomas ou eliminando-os (Akerblad, 2007; Fleck \& cols., 2003; Marques, 2000), auxiliando na manutenção e prevenção de recorrência, em qualquer ambiente de tratamento, e em pacientes com ou sem doença física concomitante (Marques, 2000).

Mesmo diante desses benefícios, a não-adesão ao tratamento para depressão é comum. Aproximadamente 1/3 dos pacientes interrompem o tratamento antidepressivo no primeiro mês e aproximadamente $45 \%$ não ultrapassam o terceiro mês de tratamento (Bultman \& Svarstad, 2000; Lin \& cols., 1995; Nemeroff, 2003).

Vários termos têm sido utilizados para denominar o quanto o paciente segue seu tratamento (aderência, concordância, adesão; em inglês: compliance, adhesion). A forma de visualização do paciente como ativo ou passivo no tratamento tem sido considerada como responsável pelas dificuldades com a terminologia (Lingam \& Scott, 2002). Muitas também são as definições desses comportamentos, entretanto, de uma forma compreensiva, conforme Milstein-Moscati, Persano e Castro (2000), a adesão à terapêutica poderia ser definida como a medida em que o comportamento do paciente coincide com a prescrição médica, tanto no aspecto farmacológico quanto no comportamental. Neste texto, optou-se pela denominação "adesão" por esta compreender uma participação mais ativa do paciente. 
Apesar da falta de consenso a respeito da terminologia e conceituação, a não-adesão ao tratamento tem sido considerada como um fenômeno universal (Lingam \& Scott, 2002). Assim, concomitantemente às preocupações de profissionais e autoridades de saúde sobre o alto consumo de medicamentos, a não-adesão ao tratamento medicamentoso tem tomado importância e sido incluída no rol de preocupações dos profissionais de saúde, juntamente com outros fatores que influenciam o uso racional de recursos terapêuticos (Leite \& Vasconcellos, 2003). Esse cuidado pode ser compreensível ao serem avaliadas as repercussões pessoais, sociais e financeiras que podem advir dessa postura do paciente frente ao seu tratamento (Colom \& Vieta, 2002; Demyttenaere, 2003; Schou, 1997).

Na tentativa de explicar, predizer e compreender suas causas, achados da literatura internacional, citados a seguir, indicam que a não-adesão ao tratamento antidepressivo estaria associada a fatores relativos aos pacientes, à doença, ao tratamento, ao profissional de saúde e ao ambiente social.

No que diz respeito aos fatores relativos ao paciente, Cramer (1998), por exemplo, verificou que pacientes mais velhos aderiam menos ao tratamento antidepressivo. Green-Henessy e Henessy (1999), ao investigarem a associação entre fatores demográficos e auto-relato de uso de medicação antidepressiva entre 1.189 americanos, verificaram que os homens foram menos aderentes ao tratamento que as mulheres. Demyttenaere (2001) verificou que questões peculiares ao gênero influenciaram a continuidade ou não do tratamento: em homens, uma significativa melhora no funcionamento ocupacional, social ou familiar aumentava o risco de desistência e em mulheres, uma significante melhoria no funcionamento familiar aumentava esse risco.

Ainda em relação ao paciente, atitudes e crenças em relação à doença depressiva e ao tratamento da mesma se relacionaram à adesão/não-adesão ao tratamento. Manber e cols. (2003), mediante a aplicação do Questionário de Percepção de Pacientes sobre Depressão, observaram que a forma como indivíduos com depressão percebiam a doença depressiva podia ser útil na predição de preferência por determinada opção de tratamento, de expectativas sobre a eficácia do tratamento, de aderência e de resposta ao tratamento. Gonzáles, Willians Júnior, Noel e Lee (2005), ao acompanharem 95 pacientes durante um ano, para os quais havia sido prescrito medicação antidepressiva, verificaram que $68 \%$ deles interromperam o tratamento e que a probabilidade de adesão era menor quando os pacientes acreditavam que não necessitavam de cuidados psiquiátricos ou que seus problemas eram pequenos.

Dentre os fatores relativos à própria doença, culpa exagerada, motivação pobre e prejuízos cognitivos foram relacionados à baixa adesão de pacientes deprimidos, conforme foi apontado por revisões de literatura realizadas por DiMatteo, Lepper e Croghan (2000) e por Demyttenaere (1997).

Em relação ao próprio tratamento, a presença de efeitos colaterais, tempo e complexidade do tratamento se constituem como fatores importantes. Efeitos colaterais como ganho de peso, disfunção sexual e alterações no sono foram associados à não-adesão (Balon, 2002; Zajecka, 2000), sendo que esses efeitos se associariam mais comumente à nãoadesão em fases iniciais do tratamento, conforme verificado por Linden, Gothe, Dittmann, Schaaf (2000) em um estudo com 15.514 pacientes tratados com fluoxetina por neuropsiquiatras. Nesse estudo, 32,9\% dos pacientes interromperam o tratamento no período de observação, sendo que 3\% desses tinham como razão os efeitos colaterais percebidos nos primeiros 15 dias e 13,2\%, os eventos adversos ocorridos nos primeiros 20 dias. Lin e cols. (1995), por sua vez, ao entrevistarem 155 pacientes no primeiro e no quarto mês após o início do uso de antidepressivos, verificaram que dos $13,3 \%$ de pacientes que reclamaram de muito sono durante o dia, $50 \%$ interromperam o tratamento no primeiro mês. Os efeitos colaterais associados à interrupção do tratamento no segundo e terceiro mês foram fadiga, visão turva, ansiedade, mudança no apetite e ganho de peso.

Fatores relacionados ao profissional de saúde, como o estilo de comunicação do médico, podem interferir na adesão ao antidepressivo, conforme avaliação de Bultman e Svarstad (2000). Essa avaliação foi feita a partir das respostas dos pacientes a uma escala, quando estes estavam iniciando o uso de medicação antidepressiva e, posteriormente, quando retornavam ao médico para dar continuidade ao tratamento. A escala se propunha a avaliar a extensão que o estilo de comunicação inicial do médico encorajava a participação do paciente no tratamento e a forma de condução do atendimento; na condução do tratamento, a escala avaliava o quanto o médico tinha um estilo de comunicação encorajadora da participação do paciente no tratamento e o quanto monitorava o uso da medicação. Os autores verificaram que um estilo de comunicação colaborativa por parte do médico melhorava o uso da medicação. Informação sobre sintomas, curso e prognóstico da doença também estiveram relacionados a adesão/ não adesão a medicamentos antidepressivos, segundo dados da revisão de Demyttenaere (1997).

Com relação ao suporte social, o encorajamento recebido de outras pessoas para que o paciente tome a medicação (Balon, 2002) e a disponibilidade da família no caso da adesão farmacológica de pacientes esquizofrênicos (Rosa, 1998) também se relacionaram com a adesão ao tratamento.

No Brasil, as taxas de não-adesão ao tratamento antidepressivo e os motivos dos pacientes para interrupção desse tipo de tratamento não têm sido muito explorados em pesquisas científicas. Entretanto, outras particularidades têm sido estudadas, como: articulações sobre diretrizes do tratamento (Fleck \& cols., 2003), etiologia da depressão (Lafer \& Vallada-Filho, 1999; Rozenthal, Laks \& Engelhardt, 2004), abordagens de tratamento como a eletroconvulsoterapia (Souza, 1999) e a estimulação magnética transcraniana (Boechat-Barros \& Brasil-Neto, 2004), e a inserção de fatores culturais envolvidos no tratamento da depressão (Martin, Quirino \& Mari, 2007).

Considerando a relevância da questão da adesão ao tratamento medicamentoso para depressão, este estudo buscou conhecer a compreensão de pacientes com transtorno depressivo unipolar, que seguiam com seu tratamento medicamentoso e outros que o haviam abandonado, a respeito de fatores que eles acreditavam facilitar e fatores que acreditavam prejudicar a adesão a esse tipo de tratamento. 


\section{Método}

\section{Participantes}

Participaram deste estudo 24 pessoas de ambos os sexos, com diagnóstico de transtorno depressivo unipolar, para os quais haviam sido prescritas medicações antidepressivas, conforme registrado nos prontuários por médicos psiquiatras do único serviço público de saúde mental (CSM) de uma cidade do Triângulo Mineiro. Os participantes foram divididos em aderentes e não aderentes ao tratamento farmacológico; tal designação se deu de forma prévia, ocorrendo mediante consulta a prontuários nos arquivos da instituição. O critério utilizado foi a assiduidade às consultas médicas nos seis meses que antecederam a realização da pesquisa.

Para serem convidados a participar no estudo, os pacientes tinham que ter pelo menos 18 anos, diagnóstico de depressão unipolar, prescrição médica de antidepressivos, registro de acompanhamento médico de no mínimo 12 meses e serem capazes de consentir com a participação. Foram excluídos deste estudo, pacientes para os quais havia registro de comorbidades psiquiátricas, num esforço de manter a homogeneidade dos grupos. A maioria dos convidados acedeu ao convite, sendo que três pessoas que não mais continuavam com o tratamento não compareceram para a entrevista, conforme haviam combinado, sem maiores explicações.

$\mathrm{O}$ grupo de aderentes ao tratamento farmacológico (GI) foi composto por 12 pacientes que realizavam tratamento exclusivamente medicamentoso e que tinham frequentado regularmente as consultas médicas nos seis meses anteriores a realização do estudo. O outro grupo (GII) foi composto por 12 pacientes que haviam realizado tratamento farmacológico para depressão por um período mínimo de 12 meses e que, sem um contrato prévio com seu médico e/ou autorização do mesmo, abandonaram o acompanhamento psiquiátrico, não mais comparecendo às consultas nos seis meses que antecederam ao período de coleta de dados (não-aderentes).

\section{Procedimento}

Utilizou-se como recurso metodológico uma entrevista semi-estruturada, realizada individualmente pela pesquisadora nas dependências da instituição ou na residência do participante, quando este assim o solicitava. As entrevistas tiveram em média 50 minutos de duração e, mediante a anuência de todos os participantes, as verbalizações foram transcritas durante a entrevista.

\section{Análise de dados}

Para análise das respostas, utilizou-se a Análise de Conteúdo, na modalidade de Análise Temática, a partir do referencial teórico de Bardin (1977). Essa técnica propõe como trabalho do analista descobrir os núcleos de sentido que compõem a comunicação e cuja presença ou frequência de aparição pode ter um significado para o objetivo analítico em pauta. As transcrições das entrevistas foram lidas em sua totalidade tanto pela pesquisadora como por dois outros psicólogos que atuaram como juizes. As seguintes etapas, propostas por Bardin, foram observadas para a realização da análise de conteúdo: leitura flutuante das entrevistas, marcação das palavras-chave (temas), nomeação dos temas e registro de frequência, e agrupamento dos temas em categorias.

As categorias identificadas em cada grupo foram então reagrupadas em três macro-categorias e enviadas a dois outros pesquisadores experientes com a técnica de análise de conteúdo para revisão de sua relevância e consistência. Esse processo resultou na formação de três perspectivas inter-relacionadas e elucidativas do problema de estudo, dando ensejo à categorização final de fatores auxiliares e dificultadores da adesão ao tratamento farmacológico para depressão.

\section{Resultados}

A consulta realizada em 2.215 prontuários possibilitou verificar que havia, entre os pacientes da instituição, $915 \mathrm{com}$ diagnóstico médico compatível com Episódio Depressivo ou Transtorno Depressivo Recorrente (CID-10). Entre estes, 0,3\% tiveram alta médica, $69 \%$ continuavam frequentando as consultas médicas e $30,7 \%$ haviam abandonado o tratamento.

Vinte e quatro pacientes responderam a entrevista que visava conhecer aspectos que eles entendiam como propiciadores e como dificultadores da adesão ao tratamento farmacológico para depressão. O GI foi composto de 12 pacientes que estavam em acompanhamento psiquiátrico e usavam medicação antidepressiva por um tempo que variava entre 12 e 36 meses (um homem e 11 mulheres, idade média de 41 anos, escolaridade média compatível com o Ensino Médio incompleto, renda familiar situada entre um e dois salários mínimos, sendo que dois afirmaram não fazer uso das medicações em conformidade com a prescrição). No GII havia 12 pacientes que abandonaram o tratamento farmacológico para depressão, realizado com médico especialista no CSM (dois homens e 10 mulheres, idade média de 40 anos, escolaridade compatível com o Ensino Médio, renda familiar aproximada de dois salários mínimos). Dois pacientes desse grupo relataram estar fazendo uso esporádico de medicação antidepressiva, fornecida sem receita médica pelo atendente da farmácia.

$\mathrm{Na}$ análise de conteúdo para os dois grupos, foram identificados 217 comentários em relação aos fatores compreendidos como facilitadores da adesão $(\mathrm{GI}=110$ e GII=107) e 198 relativos aos fatores entendidos como dificultadores da adesão (GI=98 e GII=100). Esses comentários foram agrupados em oito categorias específicas para a adesão e nove para a não-adesão. Os fatores foram semelhantes e bipolares nos dois grupos para ambos os comportamentos avaliados. Um novo agrupamento dessas categorias específicas permitiu a identificação de três aspectos centrais, que continham algumas categorias específicas exclusivas e outras polares para o comportamento de adesão/não adesão. Ou seja, a presença ou a ausência de determinado fator poderia levar à adesão ou a não adesão ao tratamento, segundo a percepção dos participantes do estudo. 
A disposição das categorias específicas dentro de cada aspecto pode ser visualizada nas tabelas 1 e 2 .

Os aspectos intrapessoais incluíram três categorias, narradas nos dois grupos como auxiliares da adesão (reconhecimento da depressão como doença, motivação do paciente para o tratamento/cura e interpretação positiva dos resultados de um tratamento), e quatro categorias, encontradas também nos dois grupos, referentes à não adesão (não reconhecimento da depressão como doença, falta de motivação para o tratamento/cura, interpretação negativa dos resultados do tratamento e sintomas da depressão, tais como desânimo intenso e prejuízos no funcionamento mental). Os aspectos interpessoais incluíram, nos dois grupos, três categorias de interferência na adesão (relação positiva médico-paciente, relação paciente-demais membros da equipe de saúde sentida como satisfatória e presença de suporte familiar) e quatro categorias de interferência na não-adesão (relação negativa médico-paciente, relação hostil entre paciente e demais membros da equipe, falta de suporte familiar e preconceito social).

O último aspecto central, contexto do tratamento, incluiu duas categorias mencionadas como facilitadores da adesão (presença de suporte material e institucional para viabilização do tratamento e acompanhamento psicológico) e apenas uma categoria para dificultadores (ausência de suporte material e institucional para viabilização do tratamento).

A Tabela 1 apresenta a frequência de temas classificados dentro de cada categoria dos aspectos intrapessoais, interpessoais e do contexto de tratamento, relativos à condições facilitadoras da adesão, para os grupos de pacientes aderentes (GI; n=110) e de pacientes não aderentes (GII; n=107) ao tratamento.

A motivação do paciente para a realização do tratamento e para cura, uma relação amistosa e de confiança com o médico e demais membros da equipe e a acessibilidade ao tratamento destacam-se nos dois grupos como fatores importantes para a adesão ao tratamento farmacológico para depressão, sendo a motivação do paciente, mais realçada pelos participantes do GI. As condições de acesso ao tratamento, representadas pela presença de suporte material e institucional para a realização do tratamento, foram mais enfatizadas pelo GII.

As categorias identificadas a partir dos discursos dos participantes dos dois grupos em relação a fatores percebidos como influências negativas à adesão podem ser visualizadas na Tabela 2. Pode-se perceber a polaridade de algumas categorias identificadas como facilitadoras da adesão, que, em seu vértice negativo ou de ausência, prejudicaria esse comportamento e o acréscimo de duas categorias relativas exclusivamente às condições propiciadoras da não adesão (sintomatologia da depressão - impacto físico e psíquico da doença, e o preconceito social em relação ao doente, à doença e ao tipo de especialidade médica). A falta de boas perspectivas no tocante ao tratamento farmacológico e as dificuldades no acesso ao tratamento foram enfatizadas pelos pacientes do GI, enquanto o não reconhecimento da depressão como uma doença, a falta de motivação para o tratamento e as dificuldades no acesso ao mesmo foram frequentes nos discursos dos pacientes do GII.

\section{Discussão}

De acordo com os dados do presente estudo, pacientes aderentes e não aderentes ao tratamento possuem percepções semelhantes em relação aos fatores relacionados à adesão e não adesão ao tratamento farmacológico para depressão, apesar de, algumas vezes, realçarem diferentemente um determinado tema

Em relação aos pacientes que haviam abandonado o tratamento no serviço gratuito, não se sabe se, entre aqueles não contatados, algum havia buscado a continuidade do tratamento no setor privado. Esses pacientes corresponde-

Tabela 1. Aspectos percebidos pelos pacientes dos grupos GI e GII como facilitadores da adesão ao tratamento medicamentoso para depressão e frequência de temas classificados dentro de cada categoria específica.

\begin{tabular}{|c|c|c|c|c|c|}
\hline \multirow{2}{*}{ Aspectos centrais } & \multirow{2}{*}{ Categorias } & \multicolumn{2}{|c|}{ G I } & \multicolumn{2}{|c|}{ GII } \\
\hline & & $\mathrm{N}$ & $\%$ & $\mathrm{n}$ & $\%$ \\
\hline \multirow{3}{*}{ Intrapessoais } & Reconhecimento da depressão como doença & 6 & 5,4 & 3 & 2,8 \\
\hline & Motivação para o tratamento/cura & 26 & 23,6 & 18 & 16,7 \\
\hline & Interpretação positiva dos resultados & 9 & 8,2 & 6 & 5,5 \\
\hline \multirow{3}{*}{ Interpessoais } & Relação positiva médico-paciente positiva & 19 & 17,3 & 20 & 18,6 \\
\hline & Relação positiva paciente-demais membros da equipe & 15 & 13,6 & 19 & 18,5 \\
\hline & Suporte familiar & 12 & 10,9 & 10 & 9,3 \\
\hline \multirow[t]{2}{*}{ Contexto de tratamento } & $\begin{array}{l}\text { Presença de suporte material e institucional para a viabilização } \\
\text { do tratamento }\end{array}$ & 17 & 15,4 & 30 & 27,7 \\
\hline & Acompanhamento psicológico & 6 & 5,4 & 1 & 0,93 \\
\hline Total & & 110 & 100 & 107 & 100 \\
\hline
\end{tabular}


Tabela 2. Aspectos percebidos pelos pacientes dos grupos GI e GII como dificultadores da adesão ao tratamento medicamentoso para depressão e frequência de temas classificados dentro de cada categoria específica.

\begin{tabular}{|c|c|c|c|c|c|}
\hline \multirow{2}{*}{ Aspectos centrais } & \multirow{2}{*}{ Categorias } & \multicolumn{2}{|c|}{ G I } & \multicolumn{2}{|c|}{ GII } \\
\hline & & $\mathrm{n}$ & $\%$ & $\mathrm{n}$ & $\%$ \\
\hline \multirow{4}{*}{ Intrapessoais } & Ausência de reconhecimento da depressão como doença & 7 & 7,2 & 12 & 12 \\
\hline & Ausência de motivação para o tratamento/cura & 8 & 8,2 & 14 & 14 \\
\hline & Interpretação negativa dos resultados & 20 & 20,6 & 6 & 6 \\
\hline & Sintomatologia & 6 & 6,2 & 6 & 6 \\
\hline \multirow{4}{*}{ Interpessoais } & Relação negativa médico-paciente & 6 & 6,2 & 9 & 9 \\
\hline & Relação negativa paciente-demais membros da equipe & 1 & 1 & 5 & 5 \\
\hline & Ausência de suporte familiar & 10 & 10,3 & 6 & 6 \\
\hline & Preconceito social & 8 & 8,3 & 6 & 6 \\
\hline Contexto de tratamento & $\begin{array}{l}\text { Ausência de suporte material e institucional para a viabilização do } \\
\text { tratamento }\end{array}$ & 32 & 32,9 & 36 & 36 \\
\hline Total & & 98 & 100 & 100 & 100 \\
\hline
\end{tabular}

ram a 30,7\% do total de pacientes da instituição consultada, confirmando as taxas de abandono à terapêutica antidepressiva verificadas nos estudos internacionais. Bultman e Svarstad (2000), Demyttenaere (2001) e Nemeroff (2003), por exemplo, verificaram que aproximadamente um entre três pacientes havia abandonado o tratamento medicamentoso antidepressivo.

Os dados obtidos mediante entrevistas possibilitaram a identificação de uma variedade de questões que os pacientes depressivos acreditam que exercem influência no seu comportamento de adesão ou de não-adesão ao tratamento medicamentoso. Esses pontos envolveram, de forma mais abrangente, questões relativas ao próprio paciente (aspectos intrapessoais), como seu desejo pelo tratamento, seu entendimento sobre a doença depressiva e sua crença em relação aos resultados do tratamento; questões referentes ao relacionamento do paciente com os profissionais da saúde, os familiares e a rede social extensa (aspectos interpessoais); e questões relativas à disponibilidade de tempo do médico, à localização do serviço, ao tempo/conveniência do paciente, à gratuidade da medicação e às condições para marcação de consultas (aspectos contextuais). Essas três perspectivas, centrais em seus pólos positivo e negativo, foram identificadas de forma similar nos dois grupos avaliados. Estudos anteriores haviam verificado que a não-adesão ao tratamento antidepressivo se trataria de um fenômeno multifatorial (Delgado, 2000; Gonzáles \& cols., 2005), o que foi confirmado com os dados do presente estudo. Bollini, Pampallona, Kupelnick, Tibaldi e Munizza (2006) consideraram, mediante revisão de estudos qualitativos referentes à temática, que se trataria de um processo complexo de comportamento, fortemente influenciado por crenças dos pacientes, ambiente que o paciente vive, prática do médico e sistema de saúde.

Com relação aos aspectos intrapessoais, segundo os participantes deste estudo, reconhecer a depressão como uma doença e distinguir seus sintomas se constituiriam em fatores auxiliares à adesão ao tratamento. Para Dowel e Hudson (1997), o processo de aceitação de um tratamento estaria intimamente ligado à aceitação do problema. Duailibi e Santos (2005) consideraram que, devido às falsas crenças de que os sintomas da depressão não são reais, muitos indivíduos não reconheciam que tinham um problema, o qual podia ser tratado, ou podiam ser desencorajados a iniciar ou se manter em tratamento por causa de vergonha e/ou preconceito.

A crença positiva e negativa em relação aos resultados advindos do tratamento medicamentoso também foi mencionada, pelos pacientes do presente estudo, como um fator que contribui para a adesão e não adesão, respectivamente. Esses resultados são similares àqueles obtidos por Gonzáles e cols. (2005) e de DiMatteo e cols. (2000), Nesses estudos, observou-se que atitudes negativas em relação à medicação, que podem se manifestar através da descrença em sua eficácia, levam os pacientes a não-adesão ao tratamento.

Os dados do presente estudo sugeriram que uma avaliação positiva dos resultados do tratamento poderia se relacionar ambiguamente com a adesão/não-adesão. Os benefícios podem disponibilizar o paciente para a continuidade de seu tratamento, o que também foi apontado por Gonzáles e cols. (2005). Esses autores verificaram que os benefícios do tratamento tornavam os pacientes mais propensos a aderirem à medicação antidepressiva ou os levava a considerarem-se curados antes da cura efetiva, o que poderia ocasionar a interrupção do tratamento (ver também Linden \& cols. 2000).

Em relação aos efeitos colaterais, na literatura eles parecem se constituir em grandes vilões à adesão ao tratamento da depressão. Autores como Lin e cols. (1995), Souza (1999), George, Peveler, Heiliger e Thompson (2000), Zajecka (2000) e Balon (2002) verificaram que esses efeitos foram causa importante, quando não principal, do abandono ao tratamento da depressão, principalmente nas primeiras se- 
manas de tratamento. Entretanto, na visão dos participantes do presente estudo, os efeitos colaterais podem se constituir em possível fator prejudicial à adesão ao tratamento, mas parecem não ser considerados como principal impeditivo, se considerarmos a frequência desse tema (apenas $4.1 \%$ dos temas referentes aos fatores prejudiciais à adesão) e de pacientes que realizaram essa avaliação (quatro participantes do GI). O tempo que tanto os pacientes aderentes como os não aderentes estavam ou estiveram em tratamento (mínimo de 12 meses), pode auxiliar na compreensão da inconsistência entre os resultados aqui obtidos e aqueles apresentados na literatura, já que esses efeitos indesjáveis acontecem principalmente nas primeiras semanas de tratamento, conforme salientado por Horimoto, Fontão e Pinto (2005).

Além, e conjuntamente às percepções a respeito da depressão e dos resultados do tratamento, os participantes deste estudo destacaram que uma alta motivação para a realização do tratamento contribui para a adesão, enquanto uma baixa motivação favorece a não adesão. Similarmente, Bultman e Svarstad (2000) apontaram a motivação como fator importante para se iniciar um tratamento, enquanto Demyttenaere (1997) relatou que pacientes não motivados tendem a não aderir ao tratamento. No presente estudo, a alta frequência de temas relacionados à motivação e também de participantes que apontaram sua importância, sugere que essa categoria deve ser considerada em estudos sobre adesão em pacientes com depressão, conforme previamente assinalado por Leite e Vasconcellos (2003). .

É importante ressaltar, conforme indicado por DiMatteo e cols. (2000), que certas características dos estados depressivos podem prejudicar a energia e a motivação dos pacientes, afetando sua disposição e também sua capacidade de seguir com o tratamento. Ou seja, a falta de motivação para o tratamento pode se dever também à própria sintomatologia da depressão, o que elevaria a importância da adesão ao tratamento neste quadro psicopatológico.

A motivação para o tratamento, de acordo com os dados levantados neste estudo, poderia ser incrementada diante da percepção pessoal de um significado existencial, ou seja, da percepção do paciente em relação à sua capacidade de auxiliar outras pessoas (e.g., cuidar dos filhos), ou ainda, de sentir-se necessário a ponto de ser alvo da preocupação de outros. Dessa forma, segundo os participantes desse estudo, a percepção do paciente quanto ao seu valor pessoal pode funcionar como estímulo para adesão ao tratamento, dado este não relatado em resultados de outras pesquisas.

Os dados deste estudo conferiram, ainda, importância às alterações do funcionamento mental do paciente e à necessidade de supervisão para efetivação do tratamento, presentes em alguns quadros depressivos, como impeditivo à realização do tratamento. Esse resultado contribui para a afirmação de Demyttenaere (1997) de que pacientes depressivos com prejuízos na concentração teriam uma maior probabilidade de não aderir ao tratamento, e confirma a preocupação de Delgado (2000) de que decisões influenciadas pelos sintomas da doença depressiva deveriam ser incluídas entre as razões para a não-adesão ao tratamento da depressão.

Em termos dos relacionamentos interpessoais, os participantes deste estudo relataram que a qualidade das relações que se estabelecem entre o paciente e os outros, considerados por ele como importantes, pode facilitar ou dificultar a adesão ao tratamento. Dentre esses "outros" destacam-se a equipe de saúde (em especial, o médico psiquiatra), a família e os amigos.

Os participantes deste estudo fizeram numerosas observações a respeito do impacto da relação médico-paciente na adesão ou não-adesão ao tratamento antidepressivo. Tanto neste estudo como em pesquisas anteriores, percebe-se que uma relação embasada na confiança técnica despertada pelo profissional e em uma postura de receptividade, cordialidade e valorização da pessoa do paciente pode funcionar como incremento positivo da adesão. DiMatteo e cols. (1993) avaliaram que a sensibilidade do médico, o tom de sua voz e a comunicação não-verbal estiveram significativamente associados à adesão de pacientes a encontros agendados. Bultman e Svarstad (2000), por sua vez, verificaram que uma postura colaborativa e de acolhimento do médico contribuía para a adesão ao tratamento e satisfação do paciente com o serviço.

Se a boa relação pode auxiliar na adesão ao tratamento, a relação negativa estabelecida com a equipe como um todo e com o médico em especial, pode atrapalhar esse processo. Os resultados deste estudo sugeriram que o médico não atencioso ou percebido como pouco amigável parece incrementar no paciente o desânimo já presente nos quadros depressivos, alimentando ainda, idéias de desvalorização pessoal, o que pode contribuir para o afastamento deste paciente do seu tratamento. A relação deficiente ou negativa com o médico também esteve relacionada a uma maior probabilidade de não-adesão ao tratamento antidepressivo nos estudos de Bultman e Svarstad (2000) e de Gonzáles e cols. (2005).

$\mathrm{O}$ atendimento prestado pela equipe de apoio, especialmente pelas recepcionistas, também foi mencionado como fator importante. A presença ou ausência de cordialidade e presteza no atendimento se relacionaria com a adesão e a não-adesão, respectivamente. De acordo com Ramos e Lima (2003), a boa recepção (ser bem tratado pela equipe, ser reconhecido por ela) está associada à denotação de boa qualidade do atendimento, o que, por sua vez, favorece a manutenção do vínculo usuário-serviço.

No contexto familiar, sentir-se acolhido, entendido e respeitado, pode funcionar como fator importante para a continuidade de um tratamento para depressão, segundo indicaram os dados deste estudo. Entretanto, o papel do suporte familiar ainda não foi arrolado entre os dados científicos a respeito na adesão de pacientes ao tratamento medicamentoso para depressão, o que contrasta com os resultados deste estudo.

Já a ausência de suporte familiar, representada neste estudo pela resistência e/ou oponência familiar ao tratamento e a falta de apoio ao paciente para a sua realização, pode confirmar a estimativa de Silva (2005) de que a ausência de participação da família prejudicaria a adesão dos pacientes ao tratamento. Autores como DiMatteo e cols. (2000) e Fawcet (1995) também incluíram a falta de ajuda e de suporte de familiares como um fator que levaria o paciente a não aderir ao tratamento.

Assim, ao paciente depressivo parece ser necessária deliberada postura de apoio familiar e social que o lembre de sua importância pessoal e das possibilidades de boas perspectivas na vida, o que proporcionaria um estímulo externo para a 
realização do tratamento. A falta de disposição, pensamentos pessimistas, diminuição da energia e cansaço "fácil", muitas vezes presentes como sintomas depressivos, podem encontrar respaldo na postura resistente ou não colaborativa de familiares. Ajuíza-se que ao paciente deprimido, que não conte com suporte familiar para a realização do tratamento, resta forte barreira a ser vencida para a realização do tratamento, necessitando o paciente de "duplo esforço" - vencer as limitações causadas pela doença e as barreiras impostas por familiares.

A percepção, pelo paciente, da existência de preconceito social em relação a doenças mentais, à função do médico especialista e aos usuários de serviços de saúde mental, foi verificada neste estudo como importante empecilho à busca e/ ou continuidade do tratamento antidepressivo. De fato, Sirey e cols. (2001) observaram que a ocorrência de desvalorização e discriminação de indivíduos que usavam serviços de saúde mental ou que possuíam uma doença mental, levou à interrupção do tratamento para depressão, especialmente em pacientes mais velhos.

É possível cogitar que o estigma a respeito do doente mental, representado por idéias e concepções preconceituosas, poderia ser incluído entre as piores facetas da doença mental em geral, causador de grandes sofrimentos e, também, conforme indicaram os dados, fator estimulador da não procura ou do abandono do tratamento, contribuindo para o agravamento da doença.

Quanto ao contexto do tratamento, foi observado um número substancial de relatos que apontavam a relevância do acesso ao tratamento para a adesão, como o oferecimento de medicação gratuita, de fácil acesso e disponibilizada no momento necessário, e o transporte para os pacientes (em especial, para aqueles que residem na zona rural, portadores de dificuldades decorrentes da própria doença e de problemas financeiros). A disponibilidade de profissionais e a oferta de vagas para consulta, de acordo com a demanda e condições/ conveniência dos pacientes, se constituiriam em fatores essenciais para se avaliar a adesão ao tratamento.

A necessidade de se investigar o acesso à medicação em pesquisas que objetivam compreender a adesão/não-adesão à terapêutica medicamentosa já havia sido discutida em trabalhos anteriores. Leite e Vasconcellos (2003), por exemplo, avaliaram que o acesso ao medicamento se constituía em problema crucial em países como o Brasil; Green-Henessy e Henessy (1999), por sua vez, ressaltaram a importância de se considerar a disponibilidade de fornecimento da medicação antidepressiva antes de avaliar seu uso. Essas considerações foram confirmadas pelos pacientes do presente estudo, em sua maioria pessoas de baixa renda, que avaliaram que as dificuldades encontradas para a obtenção da medicação, seja devido à restrições financeiras, seja pela forma utilizada pelo organismo público para disponibilizá-la ao paciente (em casos nos quais a medicação antidepressiva era oferecida gratuitamente), podem se constituir em obstáculos para a adesão.

Em relação à gratuidade e disponibilidade de antidepressivos na rede de saúde, apontada neste estudo como facilitador da adesão, não foram encontrados trabalhos nacionais com a mesma natureza, sendo possível apenas a comparação dos presentes dados com aqueles relatados em estudos interna- cionais. No entanto, ao fazer isso, deve-se considerar, com mais cuidado, as diferenças sócio-econômicas das amostras, uma vez que tais diferenças podem determinar a configuração de diferentes fatores para adesão/não-adesão ao tratamento. Kroenke e cols. (2001), por exemplo, em um estudo com a população americana, consideraram que a oferta da medicação antidepressiva poderia ter aumentado as taxas de adesão ao tratamento entre os participantes.

A localização do cuidado e as condições de acesso ao mesmo estiveram, neste e em outros trabalhos, relacionadas à adesão/não-adesão a medicações antidepressivas. Foi observado, por exemplo, que um tempo de viagem superior a 30 minutos se relacionou à não adesão ao tratamento farmacológico para depressão (Gonzáles \& cols., 2005). A localização do serviço, o tempo/conveniência do médico e do paciente e a disponibilidade de profissionais especialistas em saúde mental também foram considerados como pontos relevantes para o tratamento da depressão pelos participantes do estudo de Cooper-Patrick e cols. (1997).

Por outro lado, as dificuldades encontradas para marcação de consultas, a longa espera por atendimento, a rotatividades dos médicos e a pouca possibilidade de ter uma consulta antes do tempo previsto foram considerados como prejudiciais à adesão por parte de nossos participantes. Dunbar-Jacob (1993) havia observado, a esse respeito, que condições burocráticas de atendimento em saúde mental, as quais podem prolongar o tempo de espera do paciente, também se associaram ao comportamento de adesão/não-adesão. Vilela e Costa (1991) também chamaram atenção para o papel desestimulador da continuidade do tratamento exercido por serviços deficientes.

A respeito das deficiências encontradas nos serviços de saúde, é importante lembrar, ainda, que a carência de profissionais em psiquiatria, já considerada por Horimoto, Fontão e Pinto (2005), pode contribuir para a instalação de processos institucionais que, de alguma maneira, colaboram para o afastamento do paciente de seu tratamento. Os participantes do presente estudo apontaram a carência de profissional na instituição, a ausência do médico em datas pré-agendadas e a rotatividade de profissionais no CSM (que traz, como demanda, constante necessidade de readaptação do paciente) como agentes motivadores da interrupção do tratamento.

A relevância dada pelos participantes à psicoterapia em concomitância ao tratamento medicamentoso para a adesão ao último, está em acordo com propostas de estudos anteriores. Paykel (1995) afirmou que a colaboração entre psicoterapeutas e psiquiatras seria necessária para se atingir uma boa adesão à medicação. Pampallona, Bollini, Tibaldi, Kupelnick e Munizza (2004) verificaram que o tratamento psicológico combinado com antidepressivo esteve associado à melhoria na taxa de adesão, comparativamente ao tratamento apenas farmacológico. Lotufo Neto (2004) advertiu que a adesão ao tratamento farmacológico devia ser trabalhada em terapia. E Olfson, Marcus, Tedeschi e Wan (2006) verificaram que pacientes que receberam psicoterapia foram mais prováveis de continuar com o tratamento antidepressivo após o primeiro mês. De forma que, os dados deste estudo corroboraram aqueles da literatura ao compreenderem a importância da atuação da psicoterapia como uma intervenção capaz de colaborar com a adesão do paciente deprimido ao tratamento. 


\section{Considerações Finais}

O tema deste estudo - adesão ao tratamento farmacológico para depressão - merece atenta e continuada reflexão científica, tanto por se tratar de duas grandes questões no âmbito da saúde pública quanto pela escassez de estudos científicos nacionais a esse respeito.

Ao considerar que o paciente possui uma participação determinante no processo de um tratamento, este estudo demonstrou que a adesão e a não-adesão à terapêutica antidepressiva se constituem em fenômeno multifatorial e complementar, comportando fatores que, uma vez interrelacionados, podem conduzir a uma melhor ou pior observância das orientações relativas ao uso do medicamento antidepressivo. Assim, as duas situações avaliadas neste estudo se configuraram em processos relacionados à condições basicamente antagônicas - cuja presença ou ausência influenciam na adesão ou não ao tratamento - e, dentre esses processos, encontram-se questões intra-subjetivas, intersubjetivas e ambientais.

Considerando os três grandes aspectos inter-relacionados aos comportamentos estudados, reflete-se que a promoção da adesão ao tratamento mental deve principiar pelo reconhecimento da importância do provimento de condições básicas essenciais para efetivação de um tratamento, que poderíamos denominar "acessibilidade" ao tratamento.

Tendo observado as condições práticas de acesso ao tratamento, e assegurando-se de que estas se dêem e que ainda assim ocorra a não-adesão ao tratamento, pode-se passar à consideração de outros fatores interferentes nesse comportamento. Sob a perspectiva dos participantes deste estudo, esses outros fatores estariam associados às percepções e compreensões que o paciente faz de si mesmo, de sua doença e de seu tratamento, e também das relações que ele estabelece com seu meio e de como o meio se comporta em relação ao paciente. Considerando a perspectiva dos aspectos intra e interpessoais envolvidos nos comportamentos estudados, reflete-se como imperiosa a necessidade de estabelecer estratégias que visem ampliar e melhorar o reconhecimento que os pacientes têm da doença depressiva, do seu tratamento e dos resultados que ele pode trazer. Assim, infere-se que o uso de estratégias educativas, direcionadas a usuários de serviços de saúde mental, bem como à comunidade em geral, poderia se constituir em agente valioso para a execução de auto-avaliações mais coerentes do estado de saúde mental e necessidade de tratamento, como também para a desmistificação da loucura.

Acredita-se que os resultados deste estudo podem dar ensejo à qualificação dos profissionais que prestam serviço no CSM para que trabalhem de forma a facilitar a adesão do paciente ao tratamento. Num ângulo mais ampliado, podem subsidiar programas de formação, treinamento e capacitação de equipes de saúde mental para o manejo de pacientes com transtorno depressivo, com vistas a oferecer uma melhor qualidade de atendimento e a incrementar a possibilidade de adesão ao tratamento. Podem, também, fomentar a discussão de que os cursos de formação médica necessitam aprofundar a reflexão sobre a importância de formar profissionais atentos para a relevância que a relação médico-paciente assume no tratamento do paciente (especialmente do portador de transtorno mental), e prepará-los melhor para esse manejo. Adicionalmente, acredita-se que esse conhecimento possa vir a contribuir para a literatura específica sobre o tema, iniciando, em âmbito nacional, uma compreensão a respeito dessa importante questão, além de contribuir para geração de novas hipóteses de pesquisa.

Entretanto, a amostra composta por participantes advindos de um único centro de saúde mental limita a interpretação de resultados à própria amostra. Assim, sugere-se o desenvolvimento de pesquisas englobando um maior número de indivíduos representantes de outros contextos de atendimento psiquiátrico, com a finalidade de identificar, correlacionar e/ou confirmar variáveis envolvidas no comportamento de adesão e de não-adesão ao tratamento. Uma outra sugestão seria direcionar estudos para a construção e validação de instrumentos que indiquem a probabilidade do paciente aderir ou não ao tratamento farmacológico da depressão.

\section{Referências}

Akerblad, A C. (2007). Adherence to antidepressant medication. Tese de Doutorado, Acta Universitates Upsalienses, Faculty of Medicine, Uppsala.

Balon, R. (2002). Managing compliance. Psychiatric Times, 19(5), 1-2. Retirado em 20/01/2005, de http://www.psychiatrictimes. com/p020543.html.

Bardin, L. (1977). Análise de conteúdo. Lisboa: Martins Fontes.

Bollini, P., Pampallona, S., Kupelnick, B., Tibaldi, G., \& Munizza, C. (2006). Improving compliance in depression: a systematic review of narrative reviews. Journal of Clinical Pharmacy and Therapeutics, 31, 253-260.

Boechat-Barros, R, \& Brasil-Neto, J. P. (2004). Estimulação magnética transcraniana na depressão: resultados obtidos com duas aplicações semanais. Revista Brasileira de Psiquiatria, 26, 100-102.

Bultman, D. C., \& Svarstad, B. L. (2000). Effects of phisician communication style on client medication beliefs and adherence with antidepressant treatment. Patient Education and Counseling, 40, 173-185.

Colom, F., \& Vieta, E. (2002). Non-adherence in psychiatric disorders: Misbehavior or clinical feature? Acta Psychiatrica Scandinavica, 105, 161-163.

Cooper-Patrick,L., Powe, N. R., Jenckes, M. W., Gonzales, J. J., Levine, D. M., \& Ford, D. E. (1997). Identification of patient attitudes and preferences regarding treatment of depression. Journal of General Internal Medicine, 12(7), 431-438.

Cramer, J. A. (1998). Enhancing patient compliance in the elderly: Packaging aids and monitoring [versão eletrônica]. Drugs Aging, 12, 7-15.

Delgado, P. L.(2000). Approaches to the enhancement of patient adherence to antidepressant medication treatment [versão eletrônica]. The Journal of Clinical Psychiatry, 61, 6-9.

Demyttenaere, K. (1997). Compliance during treatment with antidepressants. Journal of Affective Disorders, 43, 27-39.

Demyttenaere, K. (2001). Compliance and acceptance in antidepressant treatment. International Journal of Psychiatry in Clinical Practice, 5, 29-35. 
Demyttenaere, K. (2003). Risck factors and predictors of compliance in depression. Europian Neuropsychopharmacology, 13, 69- 75 .

DiMatteo, M. R., Lepper, H. S., \& Croghan, T. W. (2000). Depression is a risk factor for noncompliance with medical treatment: Meta-analysis of the effects of anxiety and depression on patient adherence. Achives of Internal Medicine, 160, 2101-2107.

DiMatteo, M. R, Sherboune, C. D., Hays, R. D., Ordway, L., Kravitz, R. L., McGlynn, E. A., Kaplan, S., \& Rogers, W. H. (1993). Physicians' characteristics influence patients' adherence to medical treatment: Results from the medical outcomes study [versão eletrônica]. Health Psychology, 12(2), 93-102.

Dowel, J., \& Hudson, R. (1997). A qualitative study of medication-taking behavior in primary care. Family Practice, 14, 369-375.

Duailibi, K., \& Santos, M. E. S. B. (2005). Custos psicossociais da depressão. Em C. H. Horimoto, D. C. G. Ayache \& J. A. Souza (Orgs.), Depressão: diagnóstico e tratamento pelo clínico (pp. 151-157). São Paulo: Roca.

Dunbar-Jacob, J. (1993). Contributions to patient adherence: Is it time to share the blame? Health Psychology, 12(2), 91-92.

Fawcet, J. (1995). Compliance: Definitions and key issues [versão eletrônica]. Journal of Clinical Psychiatry, 56, 4-8.

Fleck, M. P. A., Beny, L., Sougey, E. B., Del Porto, J. A., Brasil, M. A., \& Juruena, M. F. (2003). Diretrizes da Associação Médica Brasileira para o tratamento da depressão (versão integral). Revista Brasileira de Psiquiatria, 25, 114-122.

George, C. F., Peveler, R. C., Heiliger, S., \& Thompson, C. (2000). Compliance with tricyclic antidepressants: The value of four different methods of assessment [versão eletrônica]. British Journal of Clinical Pharmacological, 50, 166-171.

Green-Hennessy, S., \& Hennessy, K. D. (1999). Demographic differences in medication use among individuals with self-reported major depression [versão eletrônica]. Psychiatry Service, 50, 257259.

Gonzáles, J., Williams Junior, J. W., Noël, P. H., \& Lee, S. (2005). Adherence to mental health treatment in primary care clinic [versão eletrônica] The Journal of the American Board of Family Practice, 18(2), 87-96.

Hexsel, A. M. (2004). Resposta antidepressiva aguda ao metilfenidato na depressão maior: ensaio clínico randomizado duplo-cego. Dissertação de Mestrado, Universidade de São Paulo, São Paulo.

Horimoto, C. H., Fontão, M. F., \& Pinto, M. A. (2005). Uso de antidepressivo pelo clínico. Em C. H. Horimoto, D. C. G. Ayache \& J. A. Souza (Orgs.), Depressão: diagnóstico e tratamento pelo clínico (pp. 117-150). São Paulo: Roca.

Kroenke, K., West, S. L., Swindle, R., Gilsenan. A., Eckerty, G. J., Dolor, R., Starg P., Zhou, X. H., Hays, R., \& Weinberger, M. (2001). Similar effectiveness of paroxetine, fluoxetine and sertraline on primary care: A randomized trial. Journal American Medicine Association, 286, 2947-2955.

Lafer, B., \& Vallada-Filho, H. P. (1999). Genética e fisiopatologia dos transtornos depressivos. Revista Brasileira de Psiquiatria, 21, 12-17.

Leite, S. N., \& Vasconcellos, M. P. C. (2003). Adesão à terapêutica medicamentosa: elementos para a discussão de conceitos e pressupostos adotados na literatura. Ciência e Saúde Coletiva, $8,775-782$.
Lima, I. V. M., Sougey, E. V., \& Vallada Filho, H. P. (2004). Farmacogenética do tratamento da depressão: busca de marcadores moleculares de boa resposta aos antidepressivos. Revista de Psiquiatria Clínica, 31, 40-43.

Lin, E. H., Von Korff, M., Katon, W., Busch; T., Simon, G. E., Walker, E., \& Robinson P. (1995). The role of the primary care physician in patients' adherence to antidepressant therapy [versão eletrônica]. Medical Care, 33, 67-74.

Linden, M., Gothe, H., Dittmann, R. W., \& Schaaf, B. (2000). Early termination of antidepressant drug treatment. Journal of Clinical Psychopharmacology, 20(5), 523-530.

Lingam, R., \& Scott, J. (2002). Treatment non-adherence in affective disorders. Acta Psychiatrica Scandinavica, 105(3), 164-172.

Lotufo Neto, F. L. (2004). Terapia comportamental cognitiva para pessoas com transtornos bipolar. Revista Brasileira de Psiquiatria, 26, 44-46.

Machado, S. C. E. P. (2003). Depressão em hospitais gerais: avaliação do desempenho de escalas de rastreamento e desenvolvimento de instrumento para uso na rotina assistencial. Tese de Doutorado, Universidade Federal do Rio Grande do Sul, Porto Alegre.

Manber, R., Chambers, A. S., Hitt, S. K., McGshueny, C., Delgado, P., \& Allen, J. J. B. (2003). Patients' perception of their depressive illness. Journal of Psychiatric Research, 37, 335-343.

Marques, M. A. S. (2000). Depressão na interação familiar: um estudo qualitativo da depressão na interação familiar. Dissertação de Mestrado, Universidade Católica Dom Bosco, Campo Grande.

Martin, D., \& Quirino J., Mari, J. (2007). Depressão entre mulheres da periferia de São Paulo. Revista de Saúde Pública, 41(4), 591-597.

Milstein-Moscati, I., Persano, S., \& Castro, L. L. C. (2000). Aspectos metodológicos e comportamentais da adesão à terapêutica. Em L. L. C. Castro (Org.), Fundamentos de farmacoepidemiologia (pp.170-180). São Paulo: AG.

Nemeroff, C. B. (2003). Improving antidepressant adherence. Journal of Clinical Psychiatry, 64, 25-30.

Olfson, M., Marcus S. C., Tedeschi, M., \& Wan G. J. (2006). Continuity of antidepressant treatment for adults with depression in the United States. American Journal of Psychiatry, 163, 101-108.

Pampallona, S., Bollini, P., Tibaldi, G., Kupelnick, B., \& Munizza, C. (2004). Combined pharmacotherapy and psychological treatment for depression: A systematic review. Archives of General Psychiatry, 61(7), 714-719.

Paykel, E. S. (1995). Psychoterapy, medication combinations, and compliance [versão eletrônica]. The Journal of Clinical Psychiatry, 56, 24-30

Ramos, D. D., \& Lima, M. A. D. S. (2003). Acesso e acolhimento aos usuários em uma unidade de saúde de Porto Alegre. Cadernos de Saúde Pública, 19, 27-34.

Rosa, M. A. (1998). Avaliação dos fatores de aderência ao tratamento medicamentoso em pacientes esquizofrênicos. Dissertação de Mestrado, Universidade de São Paulo, São Paulo.

Rozenthal, M., Laks, J., \& Engelhardt, E. (2004). Aspectos neuropsicológicos da depressão [versão eletrônica]. Revista de Psiquiatria do Rio Grande do Sul, 26, 204-212.

Schou, M. (1997). The combat of non-compliance during prophylactic lithium treatment. Acta Psychiatica Scandinava, 95(5), 361-363. 
Silva, A. G. (2005). Adesão ao tratamento da depressão. Em C. H. Horimoto, D. C. G. Ayache \& J. A. Souza (Orgs.), Depressão: diagnóstico e tratamento pelo clínico (pp. 203-211). São Paulo: Roca

Sirey, J., Bruce, M. L., Alexopoulos, G. S., Perlick, D. A., Raue, P., Friedman, S. J., \& Meyers, B. S. (2001). Perceived stigma as a predictor of treatment discontinuation in young and older outpatients with depression. The American Journal of Psychiatry, 158(3), 479-481.

Souza, F. G. M. (1999). Tratamento da depressão [versão eletrônica] Revista Brasileira de Psiquiatria, 21, 18-23.

Souza, J. A., Fontana, J. L., \& Pinto, M. A. (2005). Depressão: uma doença, várias apresentações. Em C. H. Horimoto, D. C. G. Ayache \& J. A. Souza, J. A (Orgs.), Depressão: diagnóstico e tratamento pelo clínico (pp. 1-12). São Paulo: Roca.
Vilela, J. E. M., \& Costa, D. A. (1991). Aderência terapêutica. Informação Psiquiátrica, 10, 78 - 82.

World Health Organization (2001). The world health report. Mental health: New understanding, new hope [versão eletrônica]. Genebra: Office of Publication.

Zajecka, J. M. (2000). Clinical issues in long-term treatment with antidepressants [versão eletrônica]. Journal Clinical Psychiatry, 61, 20-25.

Recebido em 15.02.07

Primeira decisão editorial em 24.07.07

Versão final em 27.07.07

Aceito em 19.06.09 